\title{
Distinguishing wet from dry age-related macular degeneration using three-dimensional computer-automated threshold Amsler grid testing
}

\author{
Craig D Robison, ${ }^{1,2}$ Renu V Jivrajka, ${ }^{1,2}$ Simon R Bababeygy, ${ }^{1,2}$ Wolfgang Fink, ${ }^{2,3,4}$ \\ Alfredo A Sadun, ${ }^{2} \mathrm{~J} \mathrm{Sebag}^{1,2}$
}

${ }^{1}$ VMR Institute, Huntington Beach, California, USA ${ }^{2}$ Doheny Eye Institute, Keck School of Medicine at the University of Southern California, Los Angeles, California, USA

${ }^{3}$ Visual and Autonomous Exploration Systems Research Laboratory, California Institute of Technology, Pasadena,

California, USA

${ }^{4}$ Department of Electrical and Computer Engineering and Biomedical Engineering, University of Arizona, Tucson, Arizona, USA

\section{Correspondence to} Dr J Sebag, VMR Institute, 7677 Center Avenue, Suite 400, Huntington Beach, CA 92647, USA;

jsebag@VMRinstitute.com

Accepted 26 December 2010 Published Online First

26 January 2011
ABSTRACT

Background/aims With the increased efficacy of current therapy for wet age-related macular degeneration (AMD), better ways to detect wet AMD are needed. This study was designed to test the ability of threedimensional contrast threshold Amsler grid (3D-CTAG) testing to distinguish wet AMD from dry AMD.

Methods Conventional paper Amsler grid and 3D-CTAG tests were performed in 90 eyes: 63 with AMD (34 dry, 29 wet) and 27 controls. Qualitative comparisons were based upon the three-dimensional shapes of central visual field (VF) defects. Quantitative analyses considered the number and volume of the three-dimensional defects. Results 25/34 (74\%) dry AMD and 6/29 (21\%) wet AMD eyes had no distortions on paper Amsler grid. Of these, $5 / 25(20 \%)$ dry and $6 / 6(100 \%)$ wet $(p=0.03)$ AMD eyes exhibited central VF defects with 3D-CTAG. Wet AMD displayed stepped defects in 16/28 (57\%) eyes, compared with only $2 / 34(6 \%)$ of dry AMD eyes $(p=0.002)$. All three volumetric indices of VF defects were two- to four-fold greater in wet than dry AMD $(p<0.006)$. 3D-CTAG had $83.9 \%$ positive and $90.6 \%$ negative predictive values for wet AMD.

Conclusions 3D-CTAG has a higher likelihood of detecting central VF defects than conventional Amsler grid, especially in wet AMD. Wet AMD can be distinguished from dry AMD by qualitative and quantitative 3D-CTAG criteria. Thus, 3D-CTAG may be useful in screening for wet AMD, quantitating disease severity, and providing a quantitative outcome measure of therapy.

\section{INTRODUCTION}

Throughout the western world, age-related macular degeneration (AMD) is the leading cause of blindness in persons older than 65 years of age. ${ }^{1-3}$ An estimated 1.75 million Americans and 3.35 million Europeans have AMD, with a prevalence of exudative AMD approaching 8.29/100 in Caucasian persons older than 80 years. ${ }^{4}$ By 2020, this number is expected to grow by more than $50 \%{ }^{4}$ AMD currently accounts for $54.4 \%$ of blindness and $22.9 \%$ of low vision among white persons, $14.3 \%$ of blindness and $14.1 \%$ of low vision among Hispanic persons, and $4.4 \%$ of blindness and $3.2 \%$ of low vision among black persons. ${ }^{5}$

Traditional screening for $\mathrm{AMD}$ has included the paper Amsler grid, despite low sensitivity and high false-negative readings, especially concerning scotomas of $6^{\circ}$ or less. ${ }^{6} 7$ With the advent of antivascular endothelial growth factor (VEGF) therapy and the prospect of other more potent treatments, there is an increased need for earlier detection of treatable disease and a way to quantitatively monitor its response to therapy. ${ }^{8-10}$

Three-dimensional computer-automated threshold Amsler grid (3D-CTAG) testing improves upon the traditional paper Amsler grid by assessing central visual fields (VF) at several contrast sensitivity levels, not just one. ${ }^{11}$ Clinical studies have found non-invasive 3D-CTAG testing to be fast $(<5 \mathrm{~min}$ per eye), easy (intuitive use of finger), accurate $\left(<1^{\circ}\right)$ and comprehensive. ${ }^{12-15}$ Conditions such as glaucoma, ocular hypertension, macular oedema and ethambutol-induced optic neuropathy have successfully been quantitatively characterised with this approach. ${ }^{11-15}$ These previous studies demonstrated a high reproducibility ( $>90 \%$ ) of 3D-CTAG detection of central VF defects in repeat testing. ${ }^{14}$ Previous studies in $\mathrm{AMD}^{16}$ have shown that the central VF defects detected with 3D-CTAG testing correspond to anatomical defects seen on fluorescein angiography. To date, however, 3D-CTAG has only qualitatively (not quantitatively) characterised non-exudative (dry) and exudative (wet) AMD. ${ }^{16}$ What has been lacking is a quantitative index of central VF defects in AMD. 3D-CTAG testing and analysis may provide a rapid, reproducible and noninvasive test useful for screening as well as monitoring patients with AMD.

This study therefore aimed to further characterise dry and wet AMD using 3D-CTAG using both qualitative as well as quantitative assessments of central VF defects. The objective was to determine the potential utility of 3D-CTAG to screen for wet $\mathrm{AMD}$ as well as provide insight as to whether this test might be used to quantitatively monitor the response to therapy. It was hypothesised that 3D-CTAG would increase the sensitivity for detection of wet $\mathrm{AMD}$ and provide quantitative indices that would distinguish wet from dry AMD.

\section{MATERIALS AND METHODS Subjects}

This cross-sectional study included 63 eyes of 43 patients with AMD. Eyes from age-matched controls without maculopathy $(n=27)$ were also tested, bringing the total study population to 90 eyes in 70 subjects. Of the 63 eyes with $\mathrm{AMD}$, there were 19 men (44\%) and 24 women (56\%), with a mean age of $77.4 \pm 10$ years (values are mean $\pm \mathrm{SD}$ ). Of these, 34 were diagnosed with dry AMD (mean age $75.9 \pm 9.5$ years) and 29 eyes were diagnosed with wet AMD (mean age $77.7 \pm 9.9$ years) $(p=0.49)$. 
Mean visual acuity (VA) in dry AMD eyes was logarithm of the minimum angle of resolution (logMAR) 0.20 (range 0.00-0.85) (Snellen 20/32), while VA in wet AMD eyes was logMAR 0.42 (range $0.00-1.60) \quad$ (Snellen 20/53) $(p=0.009)$. There were 27 control eyes without maculopathy (mean age $68.0 \pm 10.9$ years) who had a mean VA of logMAR 0.12 (range 0.00-0.40) (Snellen 20/28).

All subjects were evaluated by a single Vitreo-Retinal Surgeon (JS) using physical examination and diagnostic testing (fluorescein angiography and combined optical coherence tomography (OCT)/scanning laser ophthalmoscopy (SLO)) in each case. The clinical diagnosis for study entry was clear-cut evidence of exudative AMD (choroidal neovascularisation with clinically significant leakage in each case) or clear-cut absence of any evidence of exudative disease. No questionable cases were included.

Fundus photography and fluorescein angiography were performed (Topcon Imagenet 2000; Topcon, Tokyo, Japan) to diagnose and classify each eye as dry (drusen, chorio-retinal atrophy and pigment clumping) or wet (choroidal neovascularisation, serous elevation of the macula or retinal pigment epithelium detachment) AMD. OCT was performed with the spectral-domain combined OCT-SLO (OPKO/OTI Inc., Miami, Florida, USA) to further characterise and confirm the diagnosis. Six radial scans through the centre of the fovea were performed with additional lines through the upper and lower arcades, as well as through the optic disc.

Eyes diagnosed with geographic atrophy and disciform scarring were excluded, but previous treatments with intra-vitreal injections was not an exclusion criterion. In 20 subjects with $\mathrm{AMD}$ in both eyes, each eye was included in the appropriate diagnostic group. To determine whether 3D-CTAG might be useful as a screening test with which to distinguish between dry and wet AMD these two groups were compared.

\section{Central VF evaluations with 3D-CTAG}

Each subject was evaluated without pupil dilation after measuring best corrected VA with habitual eyewear. Refraction was not performed prior to evaluation. Conventional central VF testing employed a paper Amsler grid of white lines on a black background held at a distance of $30 \mathrm{~cm}$. The presence of either metamorphopsia or a central VF disturbance was conveyed to the evaluator and recorded. Any abnormality (distortion or scotoma) identified by the patient using their finger to outline the abnormalities directly on the touch screen was recorded.

Each 3D-CTAG contrast level represents a horizontal cut through the 'hill-of-vision' and evaluates the central $25^{\circ}$ field of vision (radially from the centre of fixation). ${ }^{17-19}$ The five individual contrast level maps are aligned along the $z$-axis (which included $5 \%, 10 \%, 20 \%, 40 \%$ and $100 \%$ contrast) by the computer program to produce a three-dimensional topographical map of the patient's contrast sensitive central VF. Testing protocol has been previously described. ${ }^{11} 1316$

Indices used for the quantitative analysis of central VF deficits included: number of defects per eye; percentage of the hill-ofvision volume lost compared with a normal/unimpaired hill-ofvision; lost area grade (LAG; scotoma area at lowest tested contrast sensitivity level/scotoma area at highest tested contrast sensitivity level) $\times$ scotoma depth (\%); preserved area grade (PAG; intact VF area at highest tested contrast sensitivity level/ intact VF area at lowest tested contrast sensitivity level) $\times$ scotoma depth (\%); and difference area grade (DAG; DAG= |PAG-LAG|), which is the absolute value of the difference between PAG and LAG as a means of expressing the symmetry between them. Evaluation of fixation was determined by the
Table 1 Sensitivity for detection of age-related macular degeneration (AMD) with traditional paper Amsler grid testing

\begin{tabular}{lccc}
\hline Paper Amsler grid & Wet AMD & Dry AMD & Total \\
\hline Visual defects & 23 & 9 & 32 \\
No visual defect & 6 & 25 & 31 \\
Total & 29 & 34 & 63 \\
Sensitivity (\%) & 79 & 26 & \\
\hline
\end{tabular}

correlation between LAG (fixation independent) and total slope (fixation dependent). Fixation was achieved with less than $5 \%$ variability between LAG and total slope $(r=0.95, p<0.001,95 \%$ CI 0.918 to 0.970 ).

VA was determined using the logMAR scale and subsequently reconverted to the corresponding Snellen fraction. For comparison of means values between dry and wet $\mathrm{AMD}$ eyes, the least square means and standard errors were obtained using generalised estimating equations (GEE) that were adjusted for the correlation between eyes within a patient. Logistic regression analyses were also modelled to adjust for repeated patient. Correlation analyses for combined wet and dry AMD eyes were performed using patient averaged data values. SAS v9.2 (SAS Institute, Cary, North Carolina, USA) was used for all analyses.

\section{RESULTS}

\section{Sensitivity of 3D-CTAG}

Paper Amsler grid testing revealed VF defects in 23/29 (79\%) wet AMD eyes and $9 / 34$ (26\%) dry AMD eyes. Among the eyes that did not demonstrate visual defects on the traditional paper Amsler grid, $6 / 6(100 \%)$ of the wet AMD eyes $(p=0.03$, signed rank sum test) and $5 / 25(20 \%)$ dry AMD eyes ( $p=0.06$, signed rank sum test) exhibited defects with 3D-CTAG. Tables 1 and 2 display the sensitivities for detection of dry and wet AMD using the traditional paper Amsler grid and the 3D-CTAG, respectively. Figure 1 compares the traditional paper Amsler grid and the 3D-CTAG with respect to test results in dry and wet AMD, revealing a greater detection rate of abnormal findings in wet $\operatorname{AMD}(29 / 29$ eyes; 100\%) and dry AMD (14/34 eyes; 41\%) using 3D-CTAG compared with paper Amsler grid testing. Control eyes without maculopathy $(n=27)$ did not have central VF defects on 3D-CTAG testing.

\section{Qualitative analysis of 3D-CTAG in dry versus wet AMD}

Dry AMD (figure 2) exhibited uniform (steep-walled; ie, same area of VF defect observed at all five contrast levels), cylindrical, three-dimensional defects in 12/14 (86\%) eyes. Partial defects (abnormalities detected at low contrast levels that are absent at higher contrast levels) were evident in the remaining 2/14 (14\%) dry AMD eyes.

Ninety-seven per cent (28/29) of wet AMD eyes displayed abnormalities at all five contrast levels. Of the 28 wet AMD eyes that had visual defects at all contrast levels, 16/28 (57\%) had larger visual defects at low levels of contrast with smaller cylindrical defects at higher levels of contrast. As shown in

Table 2 Sensitivity for detection of age-related macular degeneration (AMD) with three-dimensional contrast threshold Amsler grid (3D-CTAG) testing

\begin{tabular}{lcll}
\hline 3D-CTAG & Wet AMD & Dry AMD & Total \\
\hline Visual defect & 29 & 14 & 43 \\
No visual defect & 0 & 20 & 20 \\
Total & 29 & 34 & 63 \\
Sensitivity (\%) & 100 & 41 & \\
\hline
\end{tabular}


Sensitivity for Detection of AMD

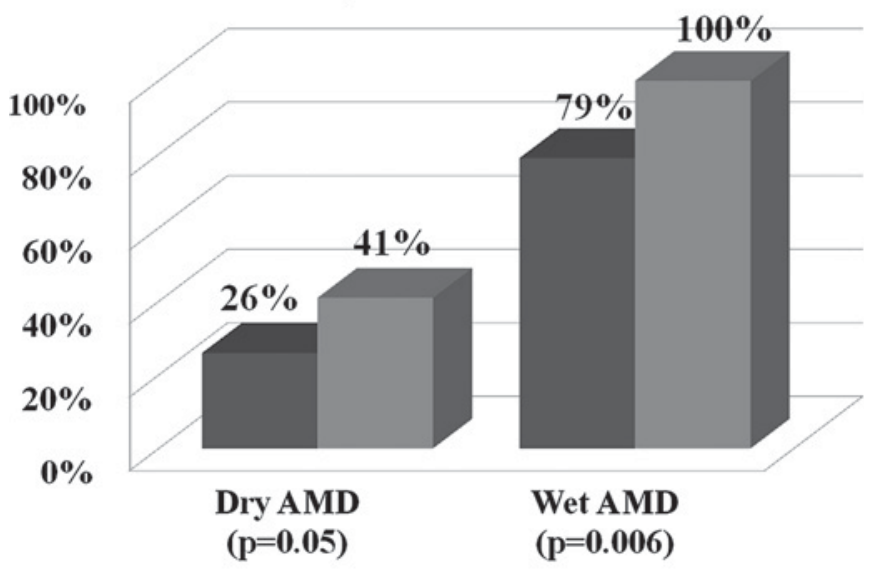

Traditional Amsler Grid Test

-3-D CTAG

Figure 1 Comparison of central visual field abnormality detection rates between traditional paper Amsler grid and the three-dimensional contrast threshold Amsler grid (3D-CTAG) in dry and wet age-related macular degeneration (AMD).

figure 3, these eyes had large central VF disturbances at low contrast levels (high contrast sensitivity) with a step-down progression to smaller cylindrical defects at increasing contrast levels (small central absolute scotoma with surrounding relative scotoma, or non-uniform cylindrical central VF abnormalities). VF defects in the remaining 12/28 (43\%) wet AMD eyes remained cylindrical at all five tested levels (16/28 vs $12 / 28$ $p=0.45, \chi^{2}$ test for equal proportions) meaning there was no increase in the area of central VF abnormalities at lower contrast levels.

\section{Quantitative analysis of 3D-CTAG in dry versus wet AMD}

One-hundred per cent of wet AMD eyes had at least one central VF defect and $14 \%$ had more than one defect. In contrast, only $41 \%$ of dry AMD eyes had a single defect, and only $3 \%$ had more than one defect. The mean number of defects observed with 3DCTAG was $0.44+0.56$ (s.d. for the upper end) and $0.44-0.44$ (s. $\mathrm{d}$. for the lower end) versus $1.24 \pm 0.79$ for wet $\mathrm{AMD}(\mathrm{p}<0.001$, GEE).

The mean LAG, which characterises the area of the threedimensional defect with respect to the lowest and highest contrast sensitivity levels, was $29.5 \%+41.7 \%$ (s.d. for the upper end) and $29.5 \%-29.5 \%$ (s.d. for the lower end) for dry AMD versus $66.7 \pm 30.9 \%$ for wet $\mathrm{AMD}(\mathrm{p}<0.001, \mathrm{GEE})$. The mean PAG, which characterises the area of the preserved VF with respect to the highest and lowest contrast sensitivity levels, was $36.2+47.5 \%$ (s.d. for the upper end) and $36.2-36.2 \%$ (s.d. for the lower end) for dry AMD versus $89.3 \pm 21.1 \%$ for wet $A M D$ $(p<0.001, G E E)$. The mean DAG, which expresses the symmetry between PAG and LAG, was $6.7+15.2 \%$ (s.d. for upper end) and $15.2-15.2 \%$ (s.d. for lower end) for dry AMD versus $22.6+26.4 \%$ (s.d. for upper end) and 22.6-22.6\% (s.d. for lower end) for wet $\operatorname{AMD}(p=0.006, \mathrm{GEE})$.

\section{Distinguishing dry AMD from wet AMD with 3D-CTAG}

Univariate stepwise logistic regression analyses were performed to distinguish wet AMD from dry AMD. The results of multiple logistic regression analysis using stepwise selection are shown in table 3. There was a high specificity for the presence of a 'step' in wet $\mathrm{AMD}$, defined as a cylindrical defect at low contrast sensitivity levels with a larger cylindrical defect at higher contrast sensitivity levels $(94.1 \%)$, and the presence of more than one 3D-CTAG defect (97.1\%). These findings each had a low sensitivity resulting in low predictive values. When stepwise selection was employed for wet $\mathrm{AMD}$, the presence of more than one defect, the presence of a 'step', and a 'hill-of-vision' volume loss of less than $2 \%$ resulted in a high sensitivity $(89.7 \%)$, specificity (85.3\%), positive predictive value (83.9\%) and negative predictive value $(90.6 \%)$.

\section{DISCUSSION}

When compared with traditional paper Amsler grid testing, 3D-CTAG had a higher likelihood of detecting central VF abnormalities in AMD. In dry AMD the sensitivity increased from $26 \%$ to $41 \%$, while in wet AMD it increased from $79 \%$ to $100 \%$. This study therefore suggests that similar to the findings in glaucoma, ${ }^{13}$ 3D-CTAG increases the ability to detect VF abnormalities in AMD compared with the traditional paper Amsler grid.

3D-CTAG detected qualitative and quantitative differences in the central VF abnormalities of patients with dry AMD compared with wet $\mathrm{AMD}$. In contrast to dry $\mathrm{AMD}$, wet $\mathrm{AMD}$ displayed a larger abnormality at lower contrast levels than at higher contrast levels. Nazemi et al ${ }^{16}$ qualitatively described dry $\mathrm{AMD}$ central visual defects as having a steeper slope and wet AMD defects as having a shallower slope, which is consistent with our findings. However, those studies did not undertake an in-depth quantitative analysis of either dry or wet AMD. As
Figure 2 Three-dimensional contrast threshold Amsler grid (3D-CTAG) plot of central visual field abnormality in dry age-related macular degeneration (AMD). A uniform (ie, same area of abnormality present at all contrast sensitivity levels) cylindrical central visual field abnormality such as this was found in 12/14 (86\%) eyes with dry AMD.

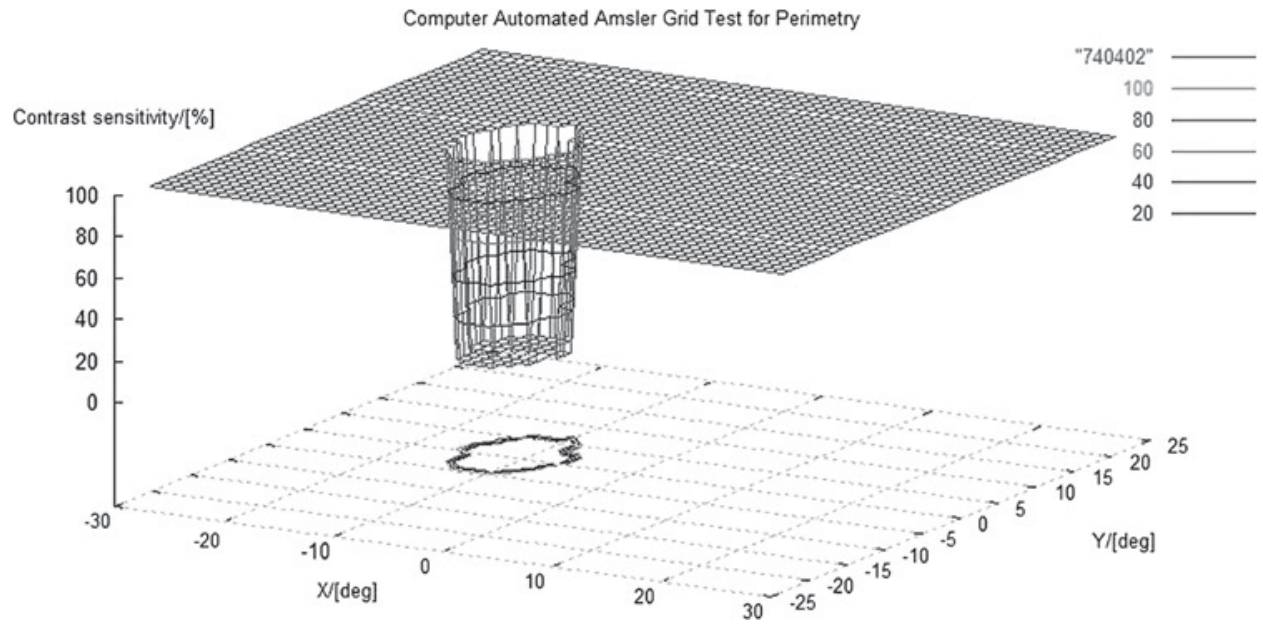


Figure 3 Three-dimensional contrast threshold Amsler grid (3D-CTAG) plot of central visual field abnormality in wet age-related macular degeneration (AMD). There is a non-uniform cylindrical abnormality; that is large area of abnormality at high contrast sensitivity levels with a distinct 'step' at lower contrast sensitivity level. This 'step' abnormality was found in 16/28 (51\%) of eyes with wet AMD.

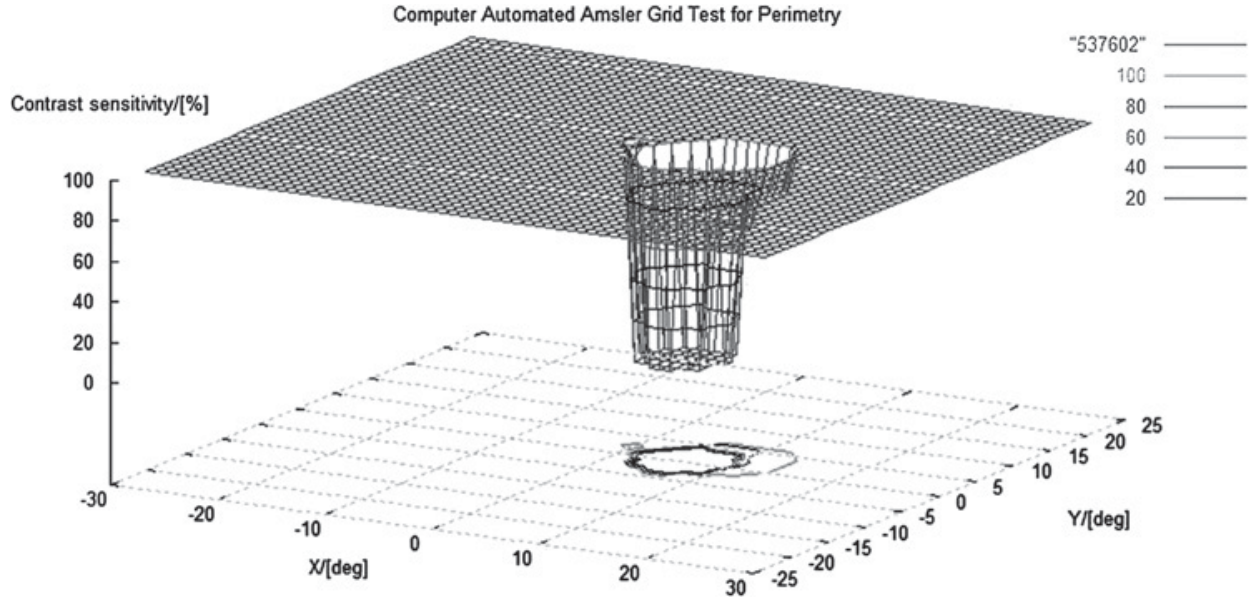

reported herein, the DAG (difference between the preserved area grade and the lost area grade) is a quantitative index of central VF defects that is useful in distinguishing wet AMD from dry AMD. As described, 3D-CTAG abnormalities in dry AMD characteristically presented as cylindrical defects, and thus had a DAG approaching zero. This was found in all dry AMD eyes tested. Wet AMD eyes demonstrated larger central VF defects at lower contrast levels, with a 'step' at intermediate and higher contrast levels. This resulted in DAG measurements in wet $\mathrm{AMD}$ eyes that were three-fold higher than in dry AMD eyes $(\mathrm{p}<0.01)$.

Since this study found a positive predictive value of $83.9 \%$ and negative predictive value of $90.6 \%$, combining the qualitative findings with quantitative indices of 3D-CTAG testing into a diagnostic algorithm for the detection of wet $\mathrm{AMD}$ has potential utility in screening for wet $\mathrm{AMD}$ in various healthcare settings. 3D-CTAG also has the benefit of ease of use with minimal technological requirements. The only task a patient is asked to perform is to trace the delineation of distortions on an Amsler grid with their index finger while maintaining fixation; a computer mouse is not involved. 3D-CTAG has the added advantage over standard-automated perimetry tests, such as the Humphrey VF analyser, of being able to utilise either central vision or peripheral vision for fixation. While it is true that AMD is likely to cause unstable fixation in standard automated perimetry devices with a central fixation marker in an otherwise isotropic hemisphere (cupola), the 3D-CTAG features the use of a rectangular screen with four distinct corners in addition to the central fixation marker. This allows the subject to maintain fixation with their peripheral, preserved vision. ${ }^{16}$

In addition, 3D-CTAG is quick and non-invasive, thus mitigating a learning effect and fatigue inherent to all VF testing. This is particularly true in patients undergoing Humphrey VF testing on both eyes, with average time of testing of up to 8 min per eye. ${ }^{20}$ Our average testing time per eye, including using the five contrast sensitive threshold values, was less than 5 min per eye.

A concern in VF testing for glaucoma is the masking of a defect secondary to the Troxler effect. However, in our previous study ${ }^{12}$ an enlarged blind spot was detected with 3DCTAG, even though the physiological blind spot is not detected in this test. Hence, despite the usual problem with Troxler suppression of arcuate scotomas in glaucoma, we are able to

Table 3 Univariate logistic regression (adjusted for the correlation between eyes within patients) for wet age-related macular degeneration (AMD) using stepwise selection

\begin{tabular}{|c|c|c|c|c|c|c|}
\hline & OR (95\% CI) & p Value & Sensitivity (\%) & Specificity (\%) & $\begin{array}{l}\text { Positive predictive } \\
\text { value }(\%)\end{array}$ & $\begin{array}{l}\text { Negative predictive } \\
\text { value }(\%)\end{array}$ \\
\hline Number of $A / G$ defects & $35.09(2.18$ to 565.1$)$ & 0.01 & - & - & - & - \\
\hline Hill-of-vision \% volume lost & 5.79 (0.003 to 9799$)$ & 0.64 & - & - & - & - \\
\hline LAG & $1.03(1.01$ to 1.04$)$ & $<0.001$ & - & - & - & - \\
\hline LAG $\geq 45.3$ & 5.44 (1.95 to 15.18$)$ & 0.001 & 72.4 & 67.6 & 65.6 & 74.2 \\
\hline PAG & $1.03(1.02$ to 1.04$)$ & $<0.001$ & - & - & - & - \\
\hline PAG $\geq 96.9 *$ & $3.99(1.43$ to 11.16$)$ & 0.008 & 69.0 & 64.7 & 62.5 & 71.0 \\
\hline DAG & $1.06(1.01$ to 1.12$)$ & 0.03 & - & - & - & - \\
\hline $\mathrm{DAG}>0 *$ & 4.16 (1.41 to 12.27$)$ & 0.01 & 62.1 & 70.6 & 64.3 & 68.6 \\
\hline Total slope & 1.03 (1.02 to 1.04$)$ & $<0.001$ & - & - & - & - \\
\hline LogMAR & 14.20 (1.79 to 112.9$)$ & 0.01 & - & - & - & - \\
\hline Scotoma shape=Step & $21.00(2.99$ to 147.6$)$ & 0.002 & 55.2 & 94.1 & 88.9 & 71.1 \\
\hline Number of defects $>1$ & $5.12(0.43$ to 61.69$)$ & 0.20 & 13.8 & 97.1 & 80.0 & 56.9 \\
\hline Step and $>1$ defect & 20.33 (4.76 to 86.73 ) & $<0.001$ & 66.5 & 91.2 & 86.4 & 75.6 \\
\hline Hill-of-vision $\%$ volume lost $>0$ and $<2$ & $9.60(2.14$ to 43.09$)$ & 0.003 & 51.7 & 88.2 & 78.9 & 68.2 \\
\hline $\begin{array}{l}\text { Step and }>1 \text { defect and hill-of-vision \% } \\
\text { volume lost }>0 \text { and }<2\end{array}$ & $51.12(10.42$ to 250.7$)$ & $<0.001$ & 89.7 & 85.3 & 83.9 & 90.6 \\
\hline
\end{tabular}

'Step' indicates a cylindrical defect at high contrast levels with a larger cylindrical defect at lower contrast levels.

*Median value of sample.

A/G, Amsler grid defect using 3D-CTAG; DAG, difference area grade; LAG, lost area grade; LogMAR, logarithm of the minimum angle of resolution; PAG, preserved area grade. 
consistently find and characterise these VF defects. It appears, therefore, that all scotomas in VF areas that are represented in the visual cortex can be detected by 3D-CTAG.

Thus, future studies should be undertaken to evaluate 3D-CTAG testing in a prospective health screening setting. Future studies could also address a potential weakness of the present study, including subjects who were either normal $(n=27)$ or had one of two diseases, that is dry $(n=34)$ or wet $(n=29) A M D$, as well as not including fellow eyes from patients with bilateral pathology. A control group with similar VA but no macular disease should also be examined. Future studies might also include other maculopathies in the study population. If these studies confirm the use of 3D-CTAG testing in AMD, then subsequent studies could use 3D-CTAG to assess the efficacy of therapy for wet AMD. Studies might also be undertaken to compare 3D-CTAG with microperimetry, which evaluates set points in the central VF, polarisation-sensitive OCT, which has shown promise in identifying retinal pigment epithelium (RPE) disease, and preferential hyperacuity perimeter, which has similarly been shown to be superior to traditional paper Amsler grid testing. ${ }^{21-24}$

Acknowledgements The authors thank Laurie Dustin, University of Southern California School of Medicine, Los Angeles, California, for performing the statistical analyses.

Funding VMR Consulting, Inc., Huntington Beach, California, USA (study design; collection, analysis and interpretation of data; writing of article; decision to submit article); NIH grant EY03040 (no involvement in study design, analysis, interpretation, writing, or decision to submit).

Competing interests WF and AAS have proprietary interests in the three-dimensional computer-automated threshold Amsler grid test described in the study as patents on the technology have been issued. All remaining authors (CDR, SRB, RVJ and JS) have no proprietary or financial interest in the manuscript.

Patient consent Obtained.

Ethics approval This study was conducted with the approval of the University of Southern California Institutional Review Board, Los Angeles, California, USA.

Contributors All authors listed have been involved in the conception and design, acquisition of data or analysis and interpretation of data, drafting the article or revising it critically for important intellectual content, and final approval of the version published.

Provenance and peer review Not commissioned; externally peer reviewed.

\section{REFERENCES}

1. Klaver CC, Wolfs RC, Vingerling JR, et al. Age-specific prevalence and causes of blindness and visual impairment in an older population: the Rotterdam Study. Arch Ophthalmol 1998;116:653-8.
2. Wang JJ, Foran S, Mitchell P. Age-specific prevalence and causes of bilateral and unilateral visual impairment in older Australians: the Blue Mountains eye study. Clin Experiment Ophthalmol 2000;28:268-73.

3. Muñoz B, West SK, Rubin GS, et al. Causes of blindness and visual impairment in a population of older Americans: The Salisbury Eye Evaluation Study. Arch Ophthalmol 2000;118:819-25.

4. Friedman DS, O'Colmain BJ, Munoz B, et al. Prevalence of age-related macular degeneration in the United States. Arch Ophthalmol 2004;122:564-72.

5. Congdon N, O'Colmain B, Klaver CC, et al. Causes and prevalence of visual impairment among adults in the United States. Arch Ophthalmol 2004;122 $477-85$.

6. Amsler M. Earliest symptoms of diseases of the macula. $\mathrm{Br} J$ Ophthalmol 1953:37:521-37.

7. Schuchard RA. Validity and interpretation of Amsler grid reports. Arch Ophthalmol 1993:111:776-80.

8. Avery RL, Pieramici DJ, Rabena MD, et al. Intravitreal bevacizumab (Avastin) for neovascular age-related macular degeneration. Ophthalmology 2006;113:363-72.

9. Rosenfeld PJ, Brown DM, Heier JS, et al. Ranibizumab for neovascular age-related macular degeneration. N Engl J Med 2006;355:1419-31.

10. Crossland $\mathbf{M}$, Rubin $\mathrm{G}$. The Amsler chart: absence of evidence is not evidence of absence. Br J Ophthalmol 2007:91:391-3.

11. Fink W, Sadun AA. Three-dimensional computer-automated threshold Amsler grid test. J Biomed Opt 2004;9:149-53.

12. Nguyen D, Fahimi A, Fink W, et al. Novel 3-D computer-automated threshold Ansler grid visual field testing of scotomas in glaucoma patients. Eur J Ophthalmol 2009; 19:776-82

13. Nazemi PP, Fink W, Sadun AA, et al. Early detection of glaucoma by means of a novel 3D computer-automated visual field test. $\mathrm{Br} \mathrm{J}$ Ophthalmol 2007:91:1331-6

14. Jivrajka RV, Kim JK, Fink W, et al. Quantitative analysis of central visual field defects in macular edema using three-dimensional computer-automated threshold Amsler grid testing. Graefes Arch Clin Exp Ophthalmol 2009;247:165-70.

15. Kim JK, Fahimi A, Fink W, et al. Characterizing ethambutol-induced optic neuropathy with a 3D computer-automated threshold Amsler grid test. Clin Experiment Ophthalmol 2008;36:484-8.

16. Nazemi PP, Fink W, Lim Jl, et al. Scotomas of age-related macular degeneration detected and characterized by means of a novel three-dimensional computerautomated visual field test. Retina 2005;25:446-53.

17. Katz J, Sommer A. Asymmetry and variation in the normal hill of vision. Arch Ophthalmol 1986;104:65-8.

18. Mills RP. Automated perimetry - Part I. J Am Intraocul Implant Soc 1984;10:347-53.

19. Schwabe R, Vonthein R, Ata N, et al. Modeling the hill of vision. In: Wall MMR, ed. Perimetry Update. New York, NY: Kugler Publications, 2001:71-9.

20. Heijl A, Ashman P. Pitfalls of automated perimetry in glaucoma diagnosis. Curr Opin Ophthalmol 1995;6:46-51.

21. Prager $\mathbf{F}$, Michels $\mathbf{S}$, Simader $C$, et al. Changes in retinal sensitivity in patients with neovascular age-related macular degeneration after systemic bevacizumab (avastin) therapy. Retina 2008;28:682-8.

22. Ahlers C, Gotzinger E, Pircher M. Imaging of the retinal pigment epithelium in age-related macular degeneration using polarization-sensitive optical coherence tomography. IOVS 2010;51:2149-57.

23. Alster $\mathbf{Y}$, Bressler NM, Bressler SB, et al. Preferential hyperacuity perimeter (PreView PHP) for detecting choroidal neovascularization study. Ophthalmology 2005;112:1758-65.

24. Goldstein $\mathbf{M}$, Loewenstein A, Barak A, et al. Results of a multicenter clinical trial to evaluate the preferential hyperacuity perimeter for detection of age-related macular degeneration. Retina 2005;25:296-303. 